\title{
Modal characteristics of a flexible cylinder in turbulent axial flow from numerical simulations
}

\author{
Jeroen De Ridder ${ }^{\mathrm{a}}$, Joris Degroote ${ }^{\mathrm{a}}$, Katrien Van Tichelen ${ }^{\mathrm{b}}$, Paul Schuurmans ${ }^{\mathrm{b}}$, Jan Vierendeels ${ }^{\mathrm{a}}$ \\ ${ }^{a}$ Department of Flow, Heat and Combustion Mechanics, Faculty of Engineering and Architecture, Ghent University, Ghent, Belgium \\ ${ }^{b}$ Belgian Nuclear Research Centre, Mol, Belgium
}

\begin{abstract}
In this article the vibration behavior of a flexible cylinder subjected to an axial flow is investigated numerically. Therefore a methodology is constructed, which relies entirely on fluid-structure interaction calculations. Consequently, no force coefficients are necessary for the numerical simulations. Two different cases are studied. The first case is a brass cylinder vibrating in an axial water flow. This calculation is compared to experiments in literature and the results agree well. The second case is a hollow steel tube, subjected to liquid lead-bismuth flow. Different flow boundary conditions are tested on this case. Each type of boundary conditions leads to a different confinement and results in different eigenfrequencies and modal damping ratios. Wherever appropriate, a comparison has been made with an existing theory. Generally, this linear theory and the simulations in this article agree well on the frequency of a mode. With respect to damping, the agreement is highly dependent on the correlation used for the normal friction coefficients in the linear theory.
\end{abstract}

Keywords: flow-induced vibrations, fluid-structure interaction, eigenmode

\section{Introduction}

Flow-induced vibrations are an important concern in the design of tube bundles. Typically, fluid-elastic instabilities occur in cross flow conditions (Pettigrew and Taylor, 2003), although axial flow conditions are also known to trigger these instabilities (Wang and $\mathrm{Ni}, 2009$ ). The dynamics of slender cylinders submerged in axial flow has already been studied for a long time: work on the added mass of two concentric cylinders dates back to Stokes (1843). The expression for the confinement effect which he derived can still be found in current text books (Chen, 1987; Païdoussis, 2004; Au-Yang, 2001).

Many of the analytical models for slender cylinders or related structures in axial flow that are used nowadays (Chen, 1987; Païdoussis, 1973; de Langre et al., 2007; Païdoussis et al., 2007; Sakuma et al., 2008; Rinaldi and Païdoussis, 2012) are mainly based on motion-induced inviscid forces (Lighthill, 1960) and are thus not based on the full Navier-Stokes equations. The effect of the viscous hydrodynamic forces is added afterwards. While the inviscid forces are derived from potential flow theory, the viscous forces are often introduced with empirical coefficients. A review on most of the available models can be found in Païdoussis (2004). The current analytical approach performs well for the frequency prediction, but the prediction of flutter type instabilities or turbulence-induced vibrations requires a good prediction of damping, which is governed by the viscous forces normal to the cylinder and thus by the mainly empirical coefficients. Therefore, research on these normal forces is still ongoing (Ersdal and Faltinsen, 2006; Divaret et al., 2012). To improve the accuracy of the predictions in annular flow, some computational research was performed as well. Belanger et al. (1994) e.g. developed a model to numerically investigate the dynamics of cylinders in laminar annular flow and Perotin and Granger (1997) developed a linear computational model for a cylinder in turbulent annular flow.

Alternative computational approaches to the coupled method, which will be used in this article, would be to either compute the viscous force coefficients required in linear theory (Phan et al., 2013; Facci and Porfiri, 2013) or directly measure modal matrices from a harmonically prescribed motion, which is typically performed for cross-flow fluidelastic instabilities. The first strategy has however the downside that all the viscous effects should be captured by the empirical formulations. The latter strategy has the disadvantage that modal matrices are determined at a fixed 
frequency of oscillation, not necessarily equal to an eigenfrequency. Modal damping is however dependent on the frequency of oscillation (Chen, 1987). Therefore, this forced displacement method should be performed with multiple frequencies. Transfer functions should be determined from these responses, followed by a determination of mass, damping and stiffness matrices if possible (Goyder, 2002). This however requires a lot of calculations.

The goal of this article is to compute modal characteristics of a single tube subjected to turbulent axial flow without empirical formulations for the viscous forces. The results for this geometry are readily compared to experimental results and existing theories. A methodology which allows the determination of modal characteristics from partitioned fluid-structure interaction (FSI) simulations will be proposed in the first part of this paper. These FSI-simulations are based on a combination of computational structural mechanics (CSM) and computational fluid mechanics (CFD). In the second part, the results of the computational method will be validated with modal characteristics of water experiments available in literature (Chen and Wambsganss, 1972). Finally the methodology will be applied to a hollow tube, vibrating in liquid $\mathrm{Pb}-\mathrm{Bi}$ eutectic. This configuration originates from the design of the MYRRHA reactor, which is currently being developed as a prototype nuclear reactor of the 4th generation (Abderrahim et al., 2010).

\section{Methodology}

In this section, it will be explained how to compute the first $N_{m}$ eigenmodes, natural frequencies and modal damping ratios of a structure in a fluid from numerical simulations. The methodology consists of several steps (see Figure 1) with a flow solver for the fluid domain around the tubes and a structural solver for the tubes themselves.

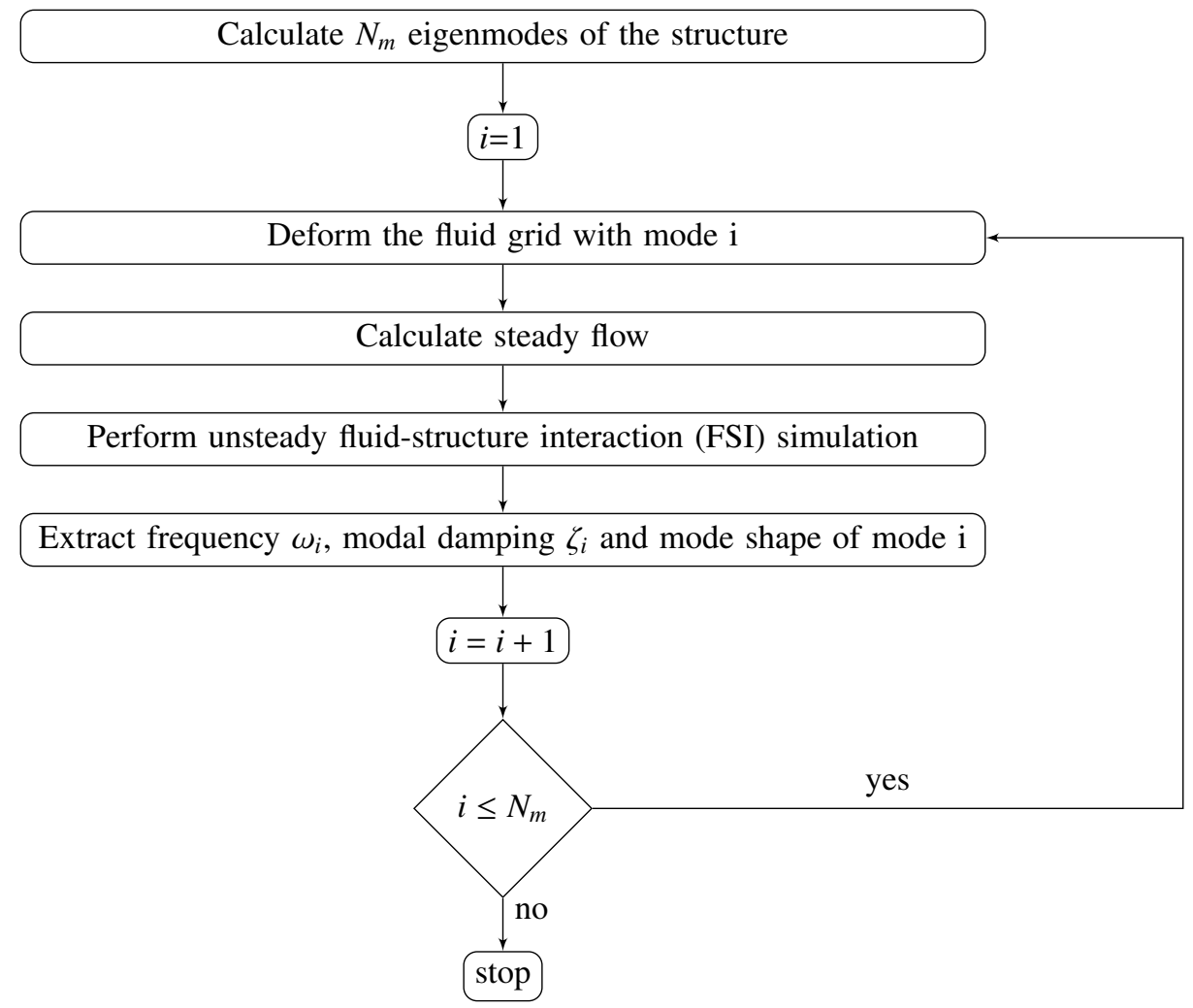

Figure 1: Simulation flowchart

Initially a finite element solver is used to solve the pure structural eigenmode problem. The damping in the structure is neglected, as, in the cases considered here, it is smaller than the damping due to the fluid; hence,

$$
\left(K-\omega_{i}^{2} M\right) \phi_{i}=0,
$$


with $K$ the stiffness matrix, $M$ the mass matrix and $\omega_{i}$ the eigenfrequency of eigenmode $\phi_{i}$. The mode shape $\phi_{i}$ is scaled such that the maximal displacement is $1(-)$. One of these mode shapes, $\phi_{i}$ is then used as a rigid wall boundary condition in a steady flow simulation. The position of the fluid-structure interface $x_{s}$ in this calculation is thus given by

$$
x_{s}=X_{s}+\alpha \phi_{i, s}
$$

where $X_{s}$ is the original position of the interface, $\phi_{i, s}$ the structural mode shape restricted to the common surface of the fluid and the structure and $\alpha$ a scaling factor. The third and final step consists of an unsteady FSI simulation, in which the free vibration of the deformed structure is computed. During these calculations both the kinematic and the dynamic equilibrium condition need to be satisfied:

$$
\begin{aligned}
& d_{s}=d_{f}, \\
& -\tau_{s} \cdot n_{s}=\tau_{f} \cdot n_{f},
\end{aligned}
$$

with $d_{s}, d_{f}$ the displacement of the interface on the structural side and on the fluid side respectively, $\tau_{f}, \tau_{s}$ the stress on the interface due to the fluid and due to the structure and $n_{s}, n_{f}$ the surface normals on the fluid-structure interface of the structural and the fluid domain. The Newtonian fluid flow itself is governed by the incompressible form of the conservation of mass and the Navier-Stokes equations, where the fluid stress tensor $\tau_{f}$ has been evaluated as $\mu_{f} \nabla \cdot \nabla v_{f}$ :

$$
\begin{aligned}
& \nabla \cdot v_{f}=0, \\
& \rho_{f}\left(\frac{\partial v_{f}}{\partial t}+v_{f} \cdot \nabla v_{f}\right)=-\nabla p+\mu_{f} \nabla \cdot \nabla v_{f}+f_{f},
\end{aligned}
$$

where $p$ stands for pressure, $v_{f}$ for fluid velocity, $\rho_{f}$ for fluid density, $\mu_{f}$ for fluid viscosity and $f_{f}$ other forces. The structure is governed by Newton's second law:

$$
\rho_{s} \frac{\partial^{2} d_{s}}{\partial t^{2}}=\nabla \cdot \tau_{s}+f_{s}
$$

with $\rho_{s}$ the solid density, $\tau_{s}$ the stress tensor and $f_{s}$ all other forces including the fluid pressure and shear stress acting on the structure. This stress tensor is determined using the constitutive equation of the material. From the FSI simulation the centerline movement of the tube is obtained. As the flow simulations are performed on structured grids, with a uniform grid spacing on the surface of the tube, the centerline displacement $d_{c l}(z)$ can be computed as

$$
d_{c l, i}(z, t)=\sum_{j} d\left(x_{j}, y_{j}, z, t\right) / N_{p}
$$

with $d(x, y, z, t)$ the displacement in a plane orthogonal to the tube axis on the outer surface of the tube and $N_{p}$ the number of points per cross section at a constant height $z$.

Generally, a free vibration can be written as a combination of modes. Therefore the $i^{\text {th }}$ mode is fitted (with removal of the lower mode contribution) according to the following expression:

$$
d_{c l, i}(z, t) \approx d_{c l, i, e s t}(z, t)=\sum_{j=1}^{i-1} \underline{g_{j}} a_{j}(z) \exp \left(-c_{j} t\right) \sin \left(\omega_{j} t+\underline{\theta_{j}}\right)+\underline{a_{i}(z)} \exp \left(-\underline{c_{i}} t\right) \sin \left(\underline{\omega_{i}} t+\underline{\theta_{i}}\right),
$$

where $g_{j}, \theta_{j}$ are the global amplitude and phase shift of the lower modes and $a_{j}(z), c_{j}, \omega_{j}$ the mode shape, damping constant and pulsation of the lower modes, determined from previous calculations and $a_{i}(z), c_{i}, \omega_{i}, \theta_{i}$ the mode shape, damping constant, pulsation and phase angle of the current mode. The underlined terms in Equation 9 are the parameters which are fitted in order to minimize the difference between the estimated centerline displacement $\left(d_{c l, i, e s t}(z, t)\right)$ and the measured centerline displacement $\left(d_{c l, i}(z, t)\right)$ :

$$
\left[g_{j}, \theta_{j}, a_{i}(z), c_{i}, \omega_{i}, \theta_{i}\right]=\arg \min _{\left[g_{j}, \theta_{j}, a_{i}(z), c_{i}, \omega_{i}, \theta_{i}\right]} \sum_{t=0}^{t_{\text {end }}}\left\|d_{c l, i}(z, t)-d_{c l, i, e s t}(z, t)\right\|_{2},
$$


with $\|\ldots\|_{2}$ the $\mathrm{L}_{2}$-norm. The modal characteristics that are reported in this paper are the natural frequency $f_{i}$ and the modal damping ratio $\zeta_{i}$ of mode $i$, such that

$$
\begin{aligned}
c_{i} & =2 \pi \zeta_{i} f_{i}, \\
\omega_{i} & =2 \pi \sqrt{1-\zeta_{i}^{2}} f_{i} .
\end{aligned}
$$

By following the procedure outlined above, the dynamic behavior of the cylinder in the axial turbulent flow is directly resolved from the coupled calculations. In this paper modal characteristics are calculated at flow velocities lower than critical flow velocities. However, the methodology could also be applied to compute the post-critical and nonlinear behavior. As it is a time-domain method, a number of initial states can be chosen and followed in time. In this way a phase-plane trajectory could be made for the coupled system. The loss of stability could then be calculated by performing simulations at different flow velocities, following an iterative procedure.

\section{Numerical methods}

There are two ways of solving an FSI-problem: either with a monolithic solver or with a partitioned approach. The advantage of the partitioned approach is that specialized solvers can be used for the different parts. The partitioned solutions can either be loosely coupled (each solver is only called once per time step) or strongly coupled (solvers are called multiple times during a time step until both the kinematic and dynamic equilibrium condition is satisfied). If the added mass effect is high, a strongly coupled scheme is necessary (Nobile et al., 2005).

The adopted coupling method is the IQN-ILS method (Interface Quasi-Newton with an approximation of the Inverse of the Jacobian from a Least-Squares approximation), which is a strongly coupled algorithm (Degroote et al., 2009). Briefly summarized, this method solves the FSI-problem with quasi-Newton iterations. As the Jacobian of the FSI-system is unknown for black-box solvers, an approximation of this Jacobian is necessary. An approximation of the inverse of the Jacobian is constructed, based on the residuals and displacements of previous coupling iterations.

The incompressible Navier-Stokes equations are solved with a finite volume solver (Fluent 14.5, Ansys). As the boundary of the fluid domain is moving, the arbitrary Lagrangian Eulerian (ALE) formulation of the Navier-Stokes equations is used. The displacements considered in this paper are on the order of $10 \mu \mathrm{m}$, so no remeshing is necessary. The displacement of the fluid grid is calculated by iteratively solving a system of linear springs between the grid nodes. The stiffness of the springs thus defines the actual displacement of the grid nodes inside the fluid domain.

The Reynolds number based on the hydraulic diameter of the simulations with lead-bismuth flow is 56000 and in the simulations with water it ranges from 127000-381000, so the flow will be turbulent. From the different approaches possible to model or to solve the turbulence in an unsteady simulation (URANS, DES, LES, DNS) the URANS methodology is adopted in this work, as the computational requirements, compared to the other methods is modest. Because of its efficient near-wall modeling the k- $\omega$ SST model of Menter (1994) is used, which blends the k- $\omega$ model near the wall with the $\mathrm{k}-\epsilon$ model in the free stream. It is known that the $\mathrm{k}-\omega$ SST will not predict flow features such as the large scale vortices typically encountered in rod bundles (Meyer, 2010). These flow instabilities are in this paper considered as an external excitation, and are thus not contributing to the intrinsic modal characteristics. To elucidate the effect of these flow instabilities on the modal characteristics, higher order models are required.

The continuity, momentum and turbulence equations are discretized with a second-order upwind scheme and a second-order time discretization is used. The solution is computed with the SIMPLE algorithm.

The structural computations are performed with a finite element solver (Abaqus 6.10, Simulia) in which the structure is modeled with second-order continuous elements. The time is discretized using the Hilber-Hughes-Taylor scheme with $\alpha=-0.41421, \beta=0.5, \gamma=0.91421$, which is a second-order time discretization. The computational cost of the structural calculation was lower than $1 \%$ of the total simulation time. Therefore, the use of beam elements for instance would only result in marginal speed gains.

\section{Modal characteristics of a flexible cylinder in water flow}

To verify the accuracy of the proposed methodology the prediction of modal characteristics of a solid brass cylinder in water flow is compared to experimental values from literature (Chen and Wambsganss, 1972). In these experiments 
Chen et al. considered a solid brass cylinder and a hollow steel cylinder in axial annular water flow. These cylinders were excited harmonically and the resulting vibration characteristics of the fundamental natural mode were then determined for different flow speeds, different hydraulic diameters and different fixations at top and bottom. The test which showed the least experimental uncertainty is computed in this article.

\subsection{Geometry and boundary conditions}

The fluid domain is an annular flow domain with a hydraulic diameter $D_{h}=0.0127 \mathrm{~m}$, in which the outer wall is kept rigid. In the experiments of Chen et al. the average water velocity was varied between 0 and $35 \mathrm{~m} / \mathrm{s}$. In the computations three different constant inlet velocities are considered: 10,20 and $30 \mathrm{~m} / \mathrm{s}$ and the properties of water: $\rho_{f}=1000 \mathrm{~kg} / \mathrm{m}^{3}, \mu_{f}=0.001$ Pa.s. As the description of the experiments does not mention any properties of the turbulence at the inlet, the turbulence inlet characteristics are chosen to be a turbulence intensity $T I=5 \%(T I=$ $\sqrt{2 k / 3} / \sqrt{v_{x}^{2}+v_{y}^{2}+v_{z}^{2}}$, where $k$ denotes the turbulent kinetic energy and $v_{x}, v_{y}, v_{z}$ the flow velocity components) and a turbulence length scale of $T L=0.1 \mathrm{~cm}$. In a different experiment with similar dimensions, designed to measure the influence of upstream turbulence, the inlet turbulence decayed after 20 times the hydraulic diameter (Mulcahy et al., 1980). Consequently, the inlet turbulence characteristics will only have a mild influence on the modal characteristics for small annuli.

The cylinder considered is made from solid brass, with a density of $\rho_{s}=8400 \mathrm{~kg} / \mathrm{m}^{3}$ and Young's modulus of elasticity $E=107 \mathrm{GPa}$. The length of the cylinder is $L=1.19 \mathrm{~m}$ and the diameter of the cylinder is $D_{c}=0.0127 \mathrm{~m}$. It is clamped at the top and at the bottom. The diameter of the outer rigid tube is $D_{o t}=0.0254 \mathrm{~m}$. Furthermore a pre-stress of $648 \mathrm{~N}$ was applied.

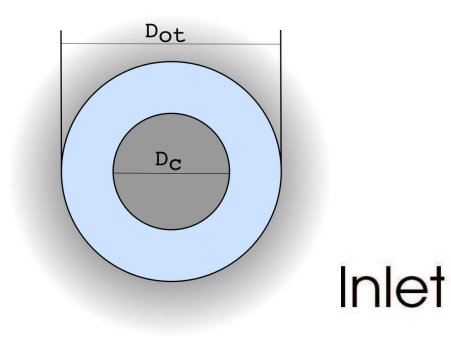

(a)

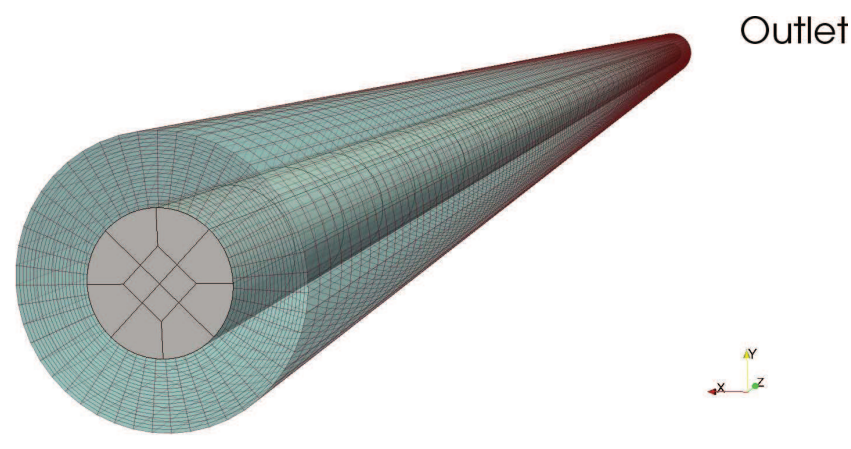

(b)

Figure 2: Geometry (2a) and computational mesh (2b) of the brass cylinder inside a water flow.

In the simulations an initial scaling factor $\alpha$ of $1 \mathrm{~mm}$ is applied. The free vibration decay is then calculated for 3 periods with 400 time steps per period.

\subsection{Comparison of modal characteristics with experiments}

Both experiments and computations predict the same trend of the natural frequency and modal damping ratio with increasing flow speed (Table 1). The natural frequency decreases slightly with increasing flow velocity. The modal damping ratio on the other hand increases significantly with higher flow speeds. The measured experimental values of the natural frequency show variations of $0.25 \mathrm{~Hz}$ and the modal damping ratio variations of 0.001 .

When the initial tensioning of the cylinders is taken into account, the computed natural frequencies agree very well with the experimental natural frequency with a maximum deviation of $3.5 \%$. The initial tension is applied by enforcing a small displacement in the structural boundary conditions. The modal damping agrees even better, with deviations in the order of the experimental uncertainty. This agreement is very important, as the current analytical methods are capable of predicting frequencies, but the damping prediction relies mostly on empirical coefficients. Therefore the presented method may lead to higher accuracy for predicting modal damping parameters, but also for a more accurate prediction of the onset of flutter instabilities. 
The exact viscosity of the water in the experiments is unknown, as the temperature is not reported. The value of the dynamic viscosity is however very temperature dependent. Therefore one calculation (with an average water velocity of $30 \mathrm{~m} / \mathrm{s}$ and an initial tension of $648 \mathrm{~N}$ ) has been done with a dynamic viscosity of $\mu=0.0009 \mathrm{~Pa}$.s as well. This decrease in viscosity corresponds to a temperature change of $5 \mathrm{~K}$ (Kestin et al., 1978). The frequency in this calculation did not change significantly and the modal damping decreased only by $2 \%$ (from 0.0303 to 0.0297 ). In URANS models, the effect of turbulence is modeled by an eddy viscosity $\mu_{t}$. The average eddy viscosity in the fluid domain changed less than $1 \%$ and is 430 times bigger than the molecular viscosity, which probably explains the small influence of the molecular viscosity on the damping.

Table 1: Experimental and computed characteristics of the fundamental mode.

\begin{tabular}{lccc}
\hline Average water velocity $(\mathrm{m} / \mathrm{s})$ & 10 & 20 & 30 \\
\hline Experimental natural frequency $(\mathrm{Hz})( \pm 0.25 \mathrm{~Hz})$ & 27.9 & 27.7 & 27.5 \\
Computed natural frequency (initial tension $=0 \mathrm{~N})(\mathrm{Hz})$ & 26.7 & 26.4 & 26.1 \\
Computed natural frequency (initial tension $=648 \mathrm{~N})(\mathrm{Hz})$ & 28.9 & 28.7 & 28.4 \\
Experimental modal damping ratio $(-)( \pm 0.001)$ & 0.013 & 0.021 & 0.030 \\
Computed modal damping ratio (initial tension $=0 \mathrm{~N})(-)$ & 0.015 & 0.024 & 0.032 \\
Computed modal damping ratio (initial tension $=648 \mathrm{~N})(-)$ & 0.014 & 0.022 & 0.030 \\
\hline
\end{tabular}

\section{Modal characteristics of a single flexible tube in turbulent $\mathrm{Pb}-\mathrm{Bi}$ flow}

As explained in the introduction, the aim of this research is to provide vibrations characteristics of a single tube in $\mathrm{Pb}-\mathrm{Bi}$ flow. The structure of this section is as follows: first the geometry, boundary conditions and material properties are discussed. After this subsection the convergence study is shown and finally the results with different flow boundary conditions are discussed.

\subsection{Geometry and boundary conditions}

Instead of considering an entire tube bundle this study focuses on one flexible empty tube within the tube bundle. Making use of the symmetry of the tube bundle, a hexagonal domain is created around one flexible tube (Figure $3 \mathrm{a}$ ). The tube bundle has a low pitch-to-diameter ratio of $P / D_{o}=1.28$ and consists of slender tubes with an aspect ratio of $L / D_{o}=237$. The empty tube considered here is a cylindrical shell, with an inner diameter of $D_{i}=5.65 \mathrm{~mm}$, an outer diameter of $D_{o}=6.55 \mathrm{~mm}$ and a length of $L=1.5 \mathrm{~m}$. The initial scaling factor $\alpha$ is set to $10 \mu \mathrm{m}$.

The computational domain at the fluid side is an annular duct, with a hexagonal cylinder at the outside and a circular cylinder at the inside. At the boundary of the annular duct both symmetric and periodic boundary conditions are considered. The periodic boundary conditions represents a situation in which all cylinders are moving in unison and thus create a periodic flow pattern. By contrast, the symmetric boundary conditions impose a zero velocity normal to the boundary and are thus very confining boundary conditions, which is the reason they are studied in this paper. It is however noted that the vibrational pattern corresponding with these boundary conditions is highly unlikely to occur in an hexagonal array of cylinders.

At the inlet a typical velocity of $1.5 \mathrm{~m} / \mathrm{s}$, with a turbulence intensity of $10 \%$ and a characteristic length scale of $0.1 \mathrm{~mm}$ is applied. The relatively high turbulent intensity of $10 \%$ is assumed because the fixations of the tube bundle at the inlet will generate turbulence. In the following paragraph it will however be shown that the influence of the turbulence parameters at the inlet is small. The Reynolds number based on the hydraulic diameter is 48000 for the periodic boundary conditions. If the symmetric boundary conditions are considered as walls, the Reynolds number is 20000, otherwise it is the same as with periodic boundary conditions.

The outlet is held at a constant pressure. The fluid is Pb-Bi eutectic which has a density of $10291 \mathrm{~kg} / \mathrm{m}^{3}$ and a dynamic viscosity of 0.0017 Pa.s. These properties correspond with a temperature of $608 \mathrm{~K}$, the average temperature in the flow channel (Sobolev, 2007).

At the bottom of the tube (at the location of the flow inlet), no nodal displacements is allowed and at the top the nodes are fixed in the $\mathrm{x}$ - and $\mathrm{y}$-direction. The resulting vibration modes are very similar to a clamped-pinned beam. The material of the tube has an elasticity modulus of $200 \mathrm{GPa}$ in the calculations and a Poisson ratio of 0.30 . 


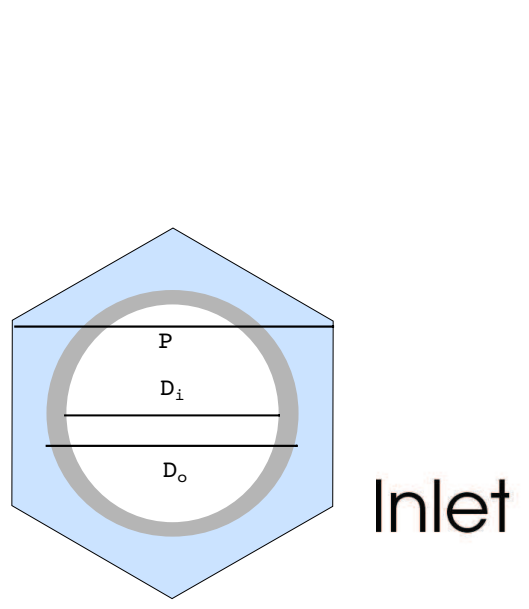

(a)

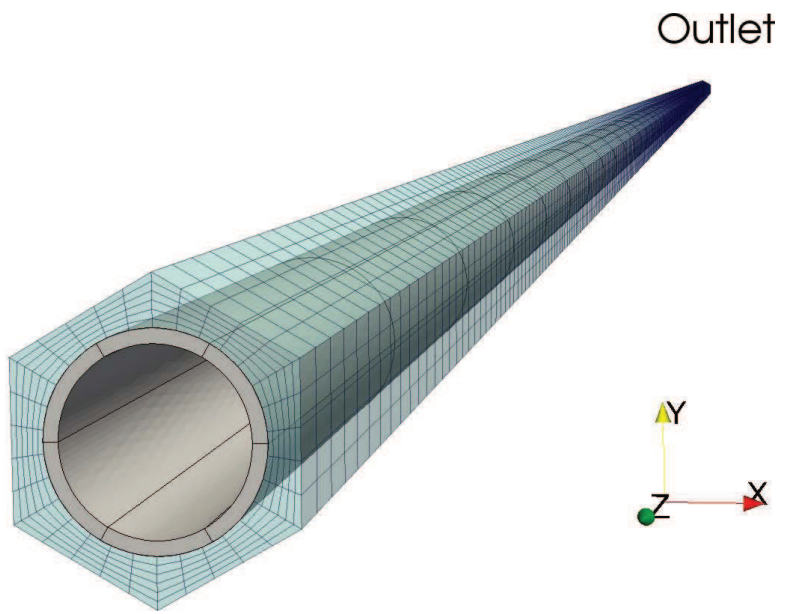

(b)

Figure 3: Cross-section of computational geometry (3a) and computational mesh (3b) with fluid area in blue and structure in grey.

\subsection{Convergence and influence of inlet conditions}

\subsubsection{Influence of grid size refinements}

Convergence of the fluid grid is tested for a tube in $\mathrm{Pb}$-Bi flow with symmetric boundary conditions at the borders of the fluid domain. Calculations with refinements in all three directions (radial, circumferential and axial) have been carried out. The different computational meshes are used to calculate the behavior of the second eigenmode of a single tube in a symmetrically confined flow with a time step $\Delta t=0.5 \mathrm{~ms}$. The first eigenmode has such a high damping that it is difficult to see the influence of the computational mesh.

From Figure 4a it can be noticed that the influence of axial refinement is small. Conversely, as the number of circumferential divisions increases from 18 to 30 , the response of the tube changes significantly. If this number is further increased to 60 only a frequency change of $2 \%$ is observed from Figure $4 \mathrm{~b}$.

In the radial direction two different types of meshes are constructed. The high Reynolds number $(\mathrm{Re}) \mathrm{mesh}$ is a hexagonal mesh with the first grid point placed in the logarithmic layer (meshes r3c30a50 and r6c30a500 in Figure 4c). The low Re mesh consists also of hexagonal cells, but with a uniform stretching applied in order to have the first grid point in the viscous sublayer (meshes r12c30a500, r18c30a500 and r24c30a500 in Figure 4c).

Within each type of grid there is only a small influence of radial refinements although the high Re meshes show an increase in damping with an increasing number of cells and the opposite is true for the low Re meshes (see Figure 4c). Further refinements of the high Re meshes are difficult because the first grid point would be in the buffer layer. As the computational cost of a high Re mesh is much lower than a low Re mesh, this type of mesh is used in the remainder of this article. The drawback is that the solution shows some dependency on the log-layer assumption. The fluid mesh that will be used in the remainder of the article consists of 6 radial , 30 circumferential and 500 axial divisions.

The finite element mesh for the structure was refined in order to achieve convergence of the frequencies of the first ten modes in vacuo. A mesh with 6 circumferential divisions, 50 axial divisions and 1 radial division is used. Further refinements of the finite element mesh showed no significant influence.

The phenomena describing the behavior of the third and fourth eigenmode require, due to the slenderness of the structure, probably the same radial and circumferential grid resolutions as the second eigenmode. Only the axial required resolution will change. However, a fluid grid with 500 axial divisions is probable fine enough, even for the fourth eigenmode.

Table 2 with the modal characteristics of the first four eigenmodes with symmetric flow boundary conditions shows that the frequency and the damping are almost insensitive to the time step size. Convergence is already achieved with 100 time steps per period. On the other hand Figure 5 shows that convergence of the modal damping in the case 


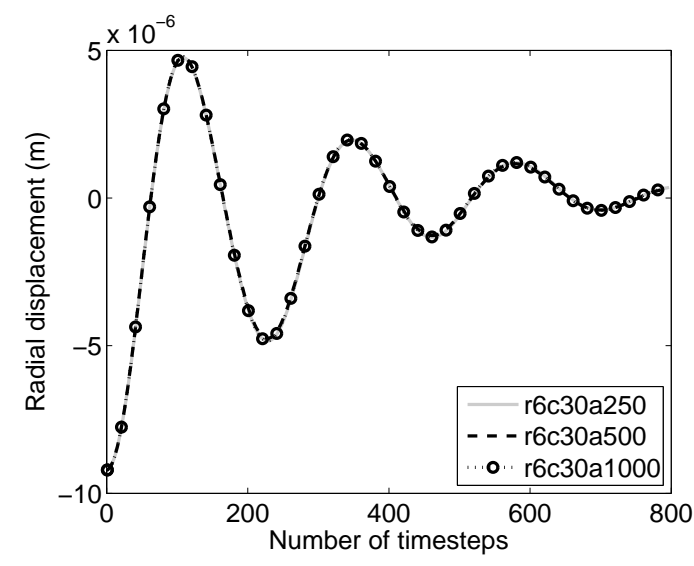

(a) Influence of the number of cells in axial direction.

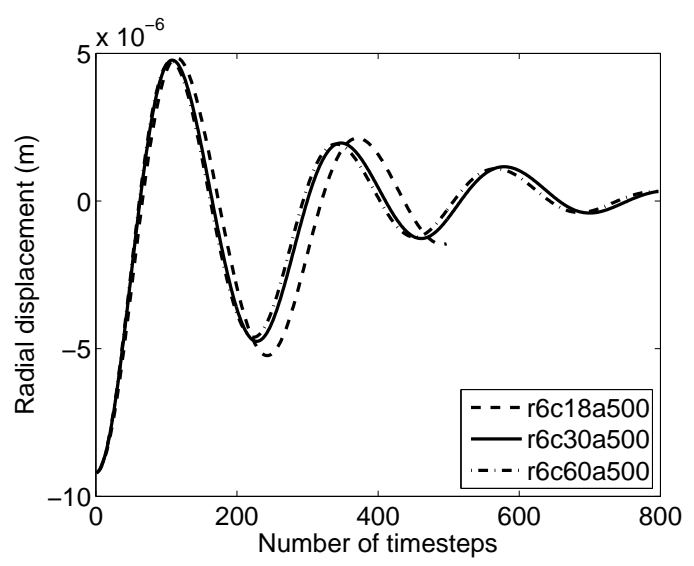

(b) Influence of the number of cells in circumferential direction.

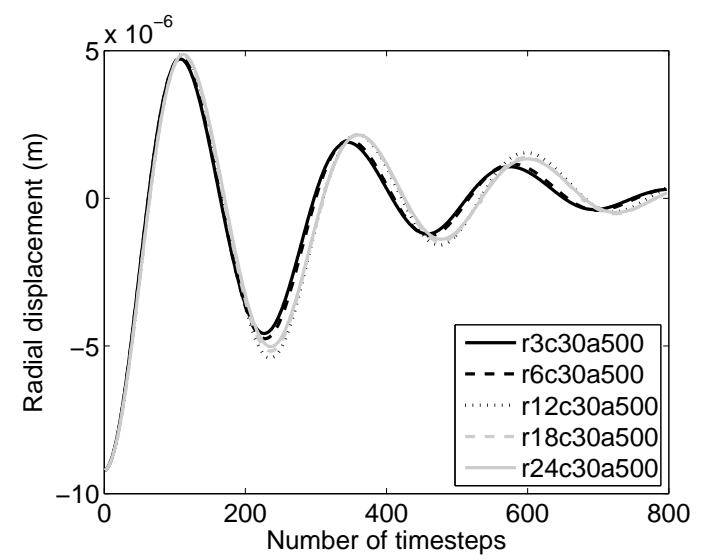

(c) Influence of the number of cells in radial direction. The first grid point of $\mathrm{r} 3 \mathrm{c} 30 \mathrm{a} 500$ and $\mathrm{r} 6 \mathrm{c} 30 \mathrm{a} 500$ is in the logarithmic layer and of $\mathrm{r} 12 \mathrm{c} 30 \mathrm{a} 500, \mathrm{r} 18 \mathrm{c} 30 \mathrm{a} 500$ and $\mathrm{r} 24 \mathrm{c} 30 \mathrm{a} 500$ in the viscous sublayer.

Figure 4: Effect of fluid grid refinement on the vibration of the second mode at $\mathrm{z}=1.2 \mathrm{~m}$. The legend denotes the number of cells in radial, circumferential and axial direction.

with periodic flow boundary conditions is much harder, as the modal damping ratio is much lower. In this case approximately 800 time steps per period are required.

\subsubsection{Influence of turbulence parameters at the inlet}

In the adopted URANS methodology the effect of turbulence is included by a turbulent (eddy) viscosity. To investigate the effect of the turbulence inlet conditions, three different steady RANS calculations have been done. The first calculation with a turbulence intensity at the inlet $\mathrm{TI}=10 \%$ and a turbulence length scale $\mathrm{TL}=0.1 \mathrm{~mm}$, the second calculation with a lower TI of $2 \%$ and the third calculation with a larger turbulence length scale, TL $=1 \mathrm{~mm}$.

The eddy viscosity is plotted along an axial line at one quarter of the flow channel radius, as shown in Figure 6. From this figure it follows that in the major part of the domain there is no difference in eddy viscosity between the different calculations. In the small region close to the inlet, the eddy viscosity shows a large variation, depending on the inlet conditions. The calculation with a turbulence intensity of $10 \%$ and a length scale of 0.1 mm produces an eddy viscosity in this region which is similar to the one in the rest of the domain. 
Table 2: Vibration characteristics with different time step sizes for the case with symmetric flow boundary conditions.

\begin{tabular}{cccc}
\hline Eigenmode & $\Delta t(\mathrm{~ms})$ & $\mathrm{f}(\mathrm{Hz})$ & $\zeta(-)$ \\
\hline 1 & 5 & 2.58 & 0.421 \\
1 & 1 & 2.56 & 0.429 \\
2 & 1 & 8.68 & 0.147 \\
2 & 0.5 & 8.64 & 0.150 \\
3 & 0.5 & 18.2 & 0.0783 \\
3 & 0.25 & 18.1 & 0.0795 \\
4 & 0.3 & 31.2 & 0.0495 \\
4 & 0.1 & 31.1 & 0.0501 \\
\hline
\end{tabular}

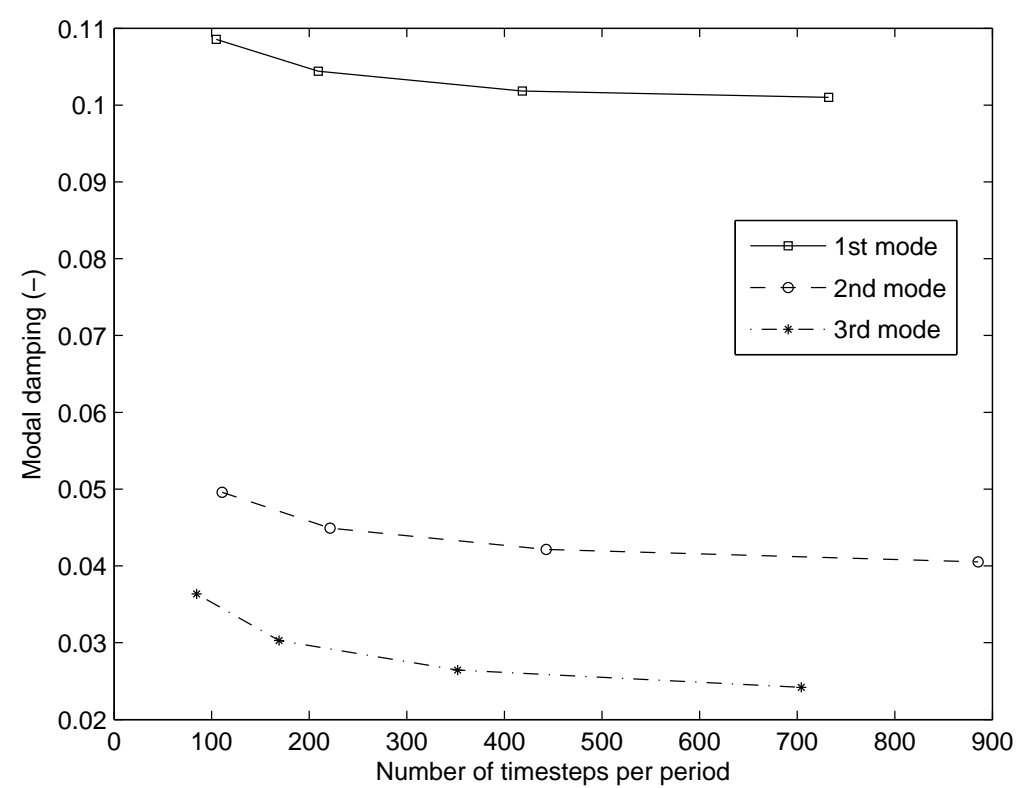

Figure 5: Influence of the number of time steps per period on the modal damping ratio for the case with periodic flow boundary conditions.

\subsection{Vibrations with symmetric boundary conditions in the flow field}

In this section the results of a flexible cylinder vibrating in $\mathrm{Pb}$ - $\mathrm{Bi}$ flow are discussed and compared to the linear theory in Païdoussis $(1973,2004)$. This theory splits the fluid forces acting on the cylinder into inviscid forces and viscous forces. The inviscid forces (normal to the surface $F_{N, i n}$ ) are determined from a momentum balance as follows:

$$
\begin{aligned}
& F_{N, i n} / L=-\left(\frac{\partial}{\partial t}+v \frac{\partial}{\partial z}\right)\left(\chi \rho_{f} A v_{y}\right), \\
& F_{N, i n} / L=-\left(\frac{\partial}{\partial t}+v \frac{\partial}{\partial z}\right)\left(\chi \rho_{f} A\left(\frac{\partial d_{c l}}{\partial t}+v \frac{\partial d_{c l}}{\partial z}\right)\right) .
\end{aligned}
$$

In this expression $v$ is the fluid velocity, $A$ is the cross-sectional area, $\chi$ is the confinement parameter, $z$ represents the axial direction and $y$ the direction of the displacement and $d_{c l}$ is the centerline displacement. The added mass for a single circular cylinder in an unconfined flow is $\rho_{f} A(C h e n, 1987)$. If the flow is confined the added mass will increase. This increase is accounted for by the confinement parameter $\chi$. The expression for the confinement parameter can be found in Chen (1987), which was derived using 2-D potential flow theory. 


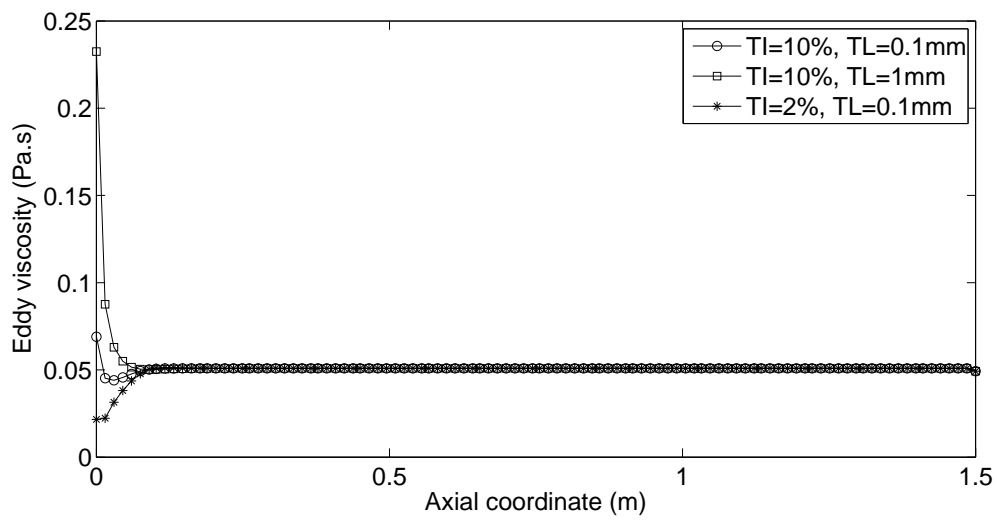

Figure 6: Influence of turbulence intensity (TI) and turbulence length scale (TL) at the inlet on the eddy viscosity along the line at one quarter of the flow channel.

The viscous forces (in the normal direction $F_{N, v i s}$ and in the longitudinal direction $F_{L, v i s}$ ) are based on the formulation of Taylor and according to Païdoussis (2004) can be linearized as

$$
\begin{aligned}
& F_{N, v i s} / L=-1 / 2 \rho_{f} D_{o} v C_{N}\left(\frac{\partial d_{c l}}{\partial t}+v \frac{\partial d_{c l}}{\partial z}\right)+1 / 2 \rho_{f} D_{o} C_{D} \frac{\partial d_{c l}}{\partial t} \\
& F_{L, v i s} / L=1 / 2 \rho_{f} D_{o} v^{2} C_{T} .
\end{aligned}
$$

In the expression for the normal forces the $C_{D}$ governs the friction due to the movement in a viscous medium without flow. This coefficient corresponds to the one Chen determined analytically for quiescent flow (Chen, 1987). In the original derivation of Taylor $C_{N}$ and $C_{T}$ should be equal. These coefficients are given by Hoerner (1965) :

$$
C_{T}=0.044\left(\rho_{f} \cdot v \cdot L / \mu_{f}\right)^{-1 / 6} .
$$

From a theoretical point of view a flow bounded by symmetric boundary conditions (slip wall) is similar to an annular flow, however without boundary layer at the outer wall. In the linear model this is implemented by defining an equivalent outer diameter $D_{e}$, such that the area of the hexagon matches the area of an equivalent circle:

$$
D_{e}=\sqrt{2 \sqrt{3} P^{2} / \pi}
$$

The linear theory is then solved by means of a Galerkin-like procedure, as explained in Païdoussis (2004). The basis functions used are the eigenmodes of a beam with pinned-clamped boundary conditions.

\subsubsection{Mode shapes}

From the numerical simulations an approximation for the mode shape in fluid flow can be constructed. This approximation can be defined as the $a_{i}(z)$ in Equation 9. Mode shapes in Pb-Bi flow or in vacuum are very similar, as can be seen in Figure 7. This observation agrees with the fact that Galerkin approaches converge with very few modes.

Although mode shapes are very similar in vacuum or in Pb-Bi flow, they are slightly shifted downstream. As the downstream shift is so small, it may be a computational artifact. However this shift is in the same direction as the fluid flow and it may be an effect of the fluid drag. The same effect is seen for very long slender structures in axial flow (de Langre et al., 2007).

\subsubsection{Vibration characteristics}

As expected from the added mass effect, the frequency of the tube vibration in $\mathrm{Pb}-\mathrm{Bi}$ flow is much lower compared to the frequency of an in vacuum vibration (see Table 3). The frequencies obtained by the linear theory or by 


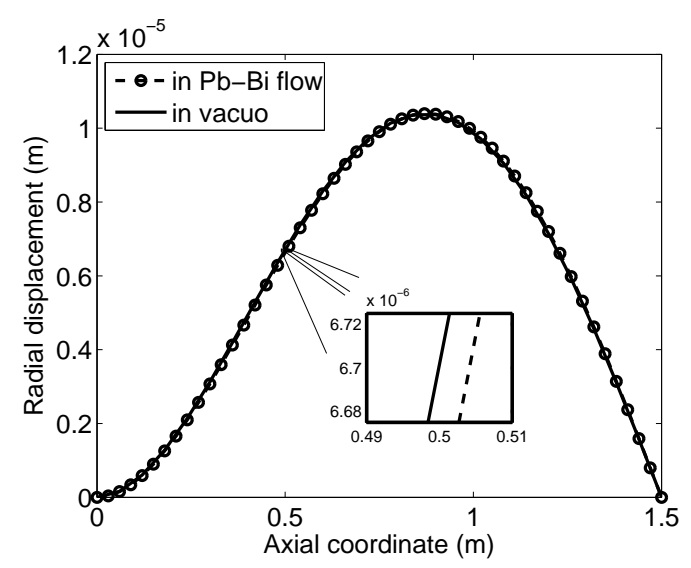

(a) First mode shape

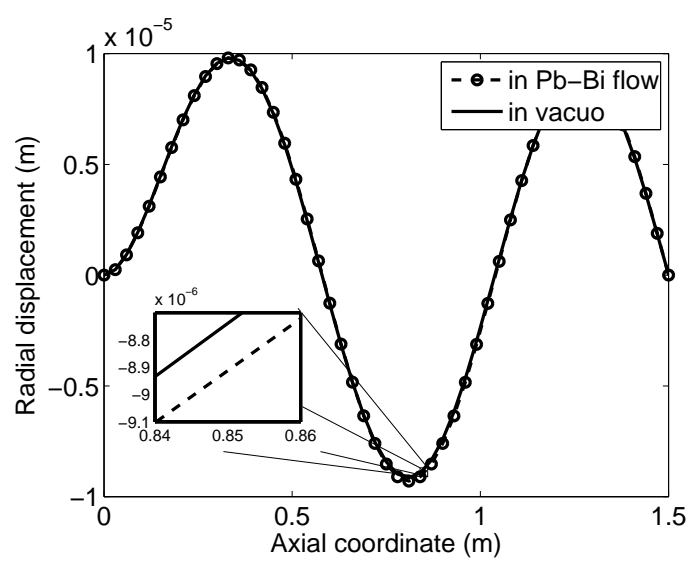

(c) Third mode shape

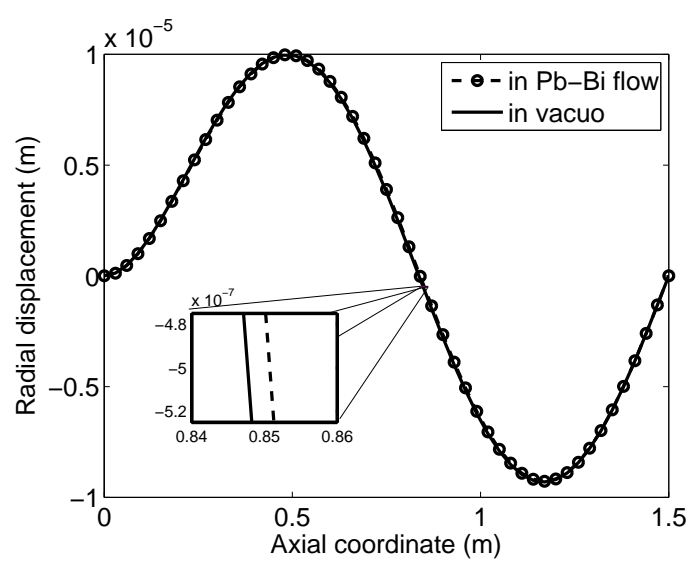

(b) Second mode shape

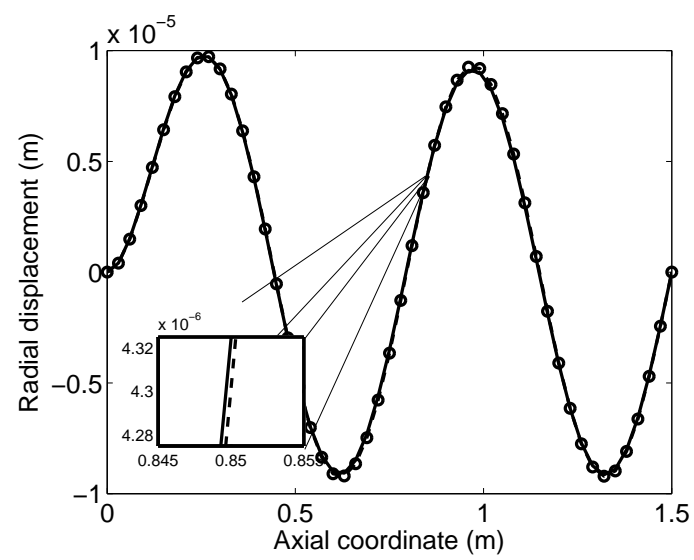

(d) Fourth mode shape

Figure 7: Mode shapes in vacuum and in $\mathrm{Pb}-\mathrm{Bi}$ flow.

the numerical simulation show an excellent agreement, which essentially means that both numeric simulations and analytical theory predict a similar added mass for the $\mathrm{Pb}-\mathrm{Bi}$ flow.

The damping predicted by the numerical solution on the other hand disagrees with the damping predicted by the theory of Païdoussis (1973). The current results predict much higher damping. A part of this disagreement may be due to the value for $C_{N}$. It is well known that the linear theory of Païdoussis (1973) underestimates the viscous effects in annular flows. The alternative theories (Mateescu and Païdoussis, 1987; Mateescu et al., 1989; Langthjem et al., 2006; Langthjem and Nakamura, 2007; Fujita and Shintani, 2001; Inada and Hayama, 1990) all assume very small flow passages, typically less than $10 \%$ of the channel diameter. Consequently, these alternative theories are not applicable as this condition is not fullfilled in this geometry, where $P / D_{o}=1.28$.

\subsection{Vibrations with periodic boundary conditions}

The second set of boundary conditions that will be applied are periodic boundary conditions. In Figure 8 the cylinder's displacement and the flow perturbation, in a plane perpendicular to the mean axial flow, due to the cylinder's vibration is shown for both the case with periodic boundary conditions and the case with symmetric boundary conditions, at approximately $0.25 T$, with $T$ the period of vibration. At $t=0$ the displacement of the tube is maximal, so at $t=0.25 T$ the velocity of the tube is maximal. 
Table 3: Vibration characteristics for the first three modes with symmetric flow boundary conditions.

\begin{tabular}{ccccccc}
\hline & $f_{1}(\mathrm{~Hz})$ & $\zeta_{1}(-)$ & $f_{2}(\mathrm{~Hz})$ & $\zeta_{2}(-)$ & $f_{3}(\mathrm{~Hz})$ & $\zeta_{3}(-)$ \\
\hline in vacuo & 11.9 & - & 38.6 & - & 80.5 & - \\
numeric & 2.56 & 0.429 & 8.64 & 0.162 & 18.1 & 0.0795 \\
linear theory & 2.51 & 0.128 & 8.56 & 0.0609 & 18.1 & 0.0385 \\
\hline
\end{tabular}

Table 4: Vibration characteristics with symmetric and periodic flow boundary conditions.

\begin{tabular}{lcccccc}
\hline & $f_{1}(\mathrm{~Hz})$ & $\zeta_{1}(-)$ & $f_{2}(\mathrm{~Hz})$ & $\zeta_{2}(-)$ & $f_{3}(\mathrm{~Hz})$ & $\zeta_{3}(-)$ \\
\hline In vacuo & 11.9 & - & 38.6 & - & 80.5 & - \\
Symmetric boundary conditions & 2.56 & 0.429 & 8.64 & 0.162 & 18.1 & 0.0795 \\
Periodic boundary conditions & 6.78 & 0.102 & 22.5 & 0.042 & 47.1 & 0.0242 \\
\hline Ratio periodic / symmetric & 2.65 & 0.233 & 2.60 & 0.271 & 2.60 & 0.304 \\
Ratio symmetric / in vacuo & 0.215 & - & 0.224 & - & 0.225 & - \\
Ratio periodic / in vacuo & 0.570 & - & 0.583 & - & 0.585 & - \\
\hline
\end{tabular}

Due to the confinement, a higher amount of fluid needs to be accelerated to higher relative velocities (relative to the velocity of the cylinder) in the case with symmetric boundary conditions compared to periodic boundary conditions. The case with symmetric boundary conditions thus has a higher inertia. The undamped natural frequency is given by $\omega_{n}^{2}=k /\left(m+m_{a}\right)$, where $k$ and $m$ are the stiffness and mass of the cylinder and $m_{a}$ is the added mass. As $\omega_{n}$ decreases with increasing added mass, the natural frequency of the case with symmetric boundary conditions will be smaller compared to the case with periodic flow boundary conditions.

Similarly, the maximum in-plane flow velocity, normalized by the speed of the cylinder, during the free vibration with symmetric boundary conditions is twice as high as during the free vibration with periodic boundary conditions and has the opposite sign compared to the movement of the cylinder. As a result, the modal damping will be higher in the case with symmetric boundary conditions.

The decrease in natural frequency can be seen in Table 4. The ratio of the frequency of the vibration with the flow field having periodic boundary conditions to the one with symmetric boundary conditions is seen to decrease slightly from the first eigenmode to the second eigenmode, while the ratio remains constant from the second to the third mode. According to Chen (1987), for a given confinement, the added mass coefficient will decrease with increasing Stokes number until it reaches a constant value. This decrease is however larger for less confined flows, which explains the observed trends in Table 4.

The same author also gives upper and lower bounds of fluid added mass (based on potential flow theory) in tube arrays. For the given configuration $\left(P / D_{o}=1.28\right)$ the ratio of maximum added mass over minimum added mass is approximately 9 . The frequency ratio of the corresponding upper and lower limit is approximately 3 , which is very close to the difference in frequency between the case with periodic boundary conditions and with symmetric boundary conditions, maybe suggesting that these two boundary conditions provide upper and lower limits for the resonance spectra of a tube bundle.

The modal damping ratio in Table 4 is smaller for the case with periodic boundary conditions than for the case with symmetric boundary condition, which is in line with the expressions of Chen (1987). In quiescent flow the modal damping ratio predicted by Sinyavskii et al. (1980) is inversely proportional to the square root of the natural frequency. In this case, both the computations with symmetric and periodic boundary conditions predict lower powers $(-0.83$ and -0.74), as can be seen in Figure 9. This corresponds however with the expressions of Chen, where this power decreases with increasing confinement.

\section{Modal characteristics of a single flexible tube in a rigid array}

Besides the symmetric and periodic boundary conditions, a third way to model the influence of the surrounding tubes, without explicitly considering them as flexible, is to include them as rigid objects in the simulation. Figure 10 displays the fluid mesh considering $3(1+6 \cdot 1 / 3)$ and $12(1+6 \cdot 1+6 \cdot 1 / 2+6 \cdot 1 / 3)$ tubes. At the boundary of the fluid 


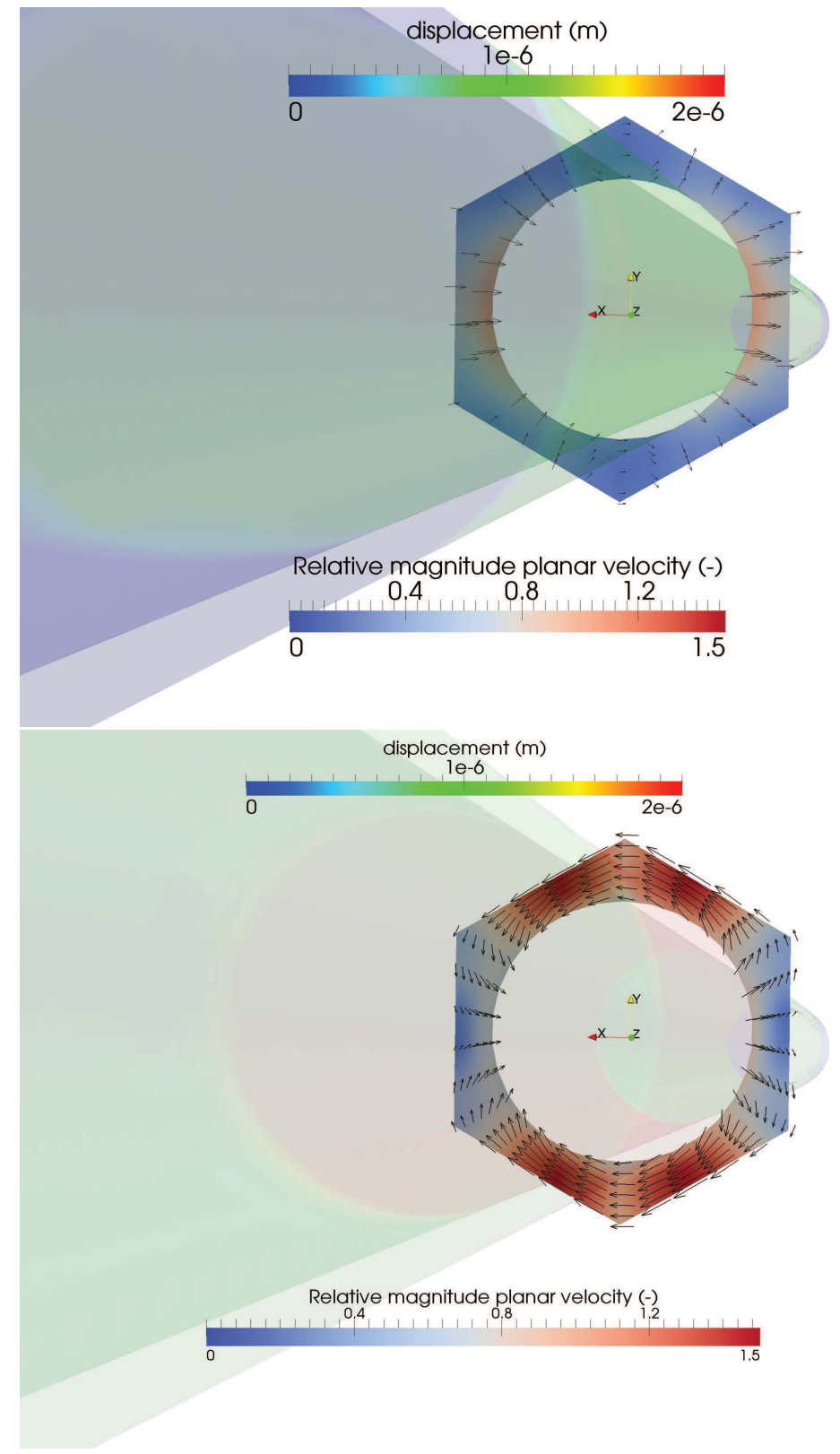

Figure 8: Isometric view of the tube, during a first-mode vibration, and the corresponding planar fluid velocity vectors. The fluid velocity vectors are scaled by the speed of the tube and are drawn in a plane halfway along and orthogonal to the tube. Both upper and lower figure are taken at $t \approx T / 4$. The top figure has periodic boundary conditions at the fluid boundaries while the bottom figure has symmetric boundary conditions.

domain periodic conditions are applied, such that the opposing sides of the surrounding hexagon are assumed periodic. The middle cylinder is taken flexible and the vibration characteristics are determined using the same methodology as in the previous sections. All dimensions and material parameters are also the same as in the previous section. The Reynolds number is 48000 , equal to the one for periodic boundary conditions.

Considering the case with two neighboring rigid tubes one immediately notes from Figure 11 that a part of the displaced fluid goes around the flexible cylinder and that another part goes through the periodic boundary, between two neighboring tubes. In the flow pattern low speed regions on the surrounding tubes appear. Half of them are 


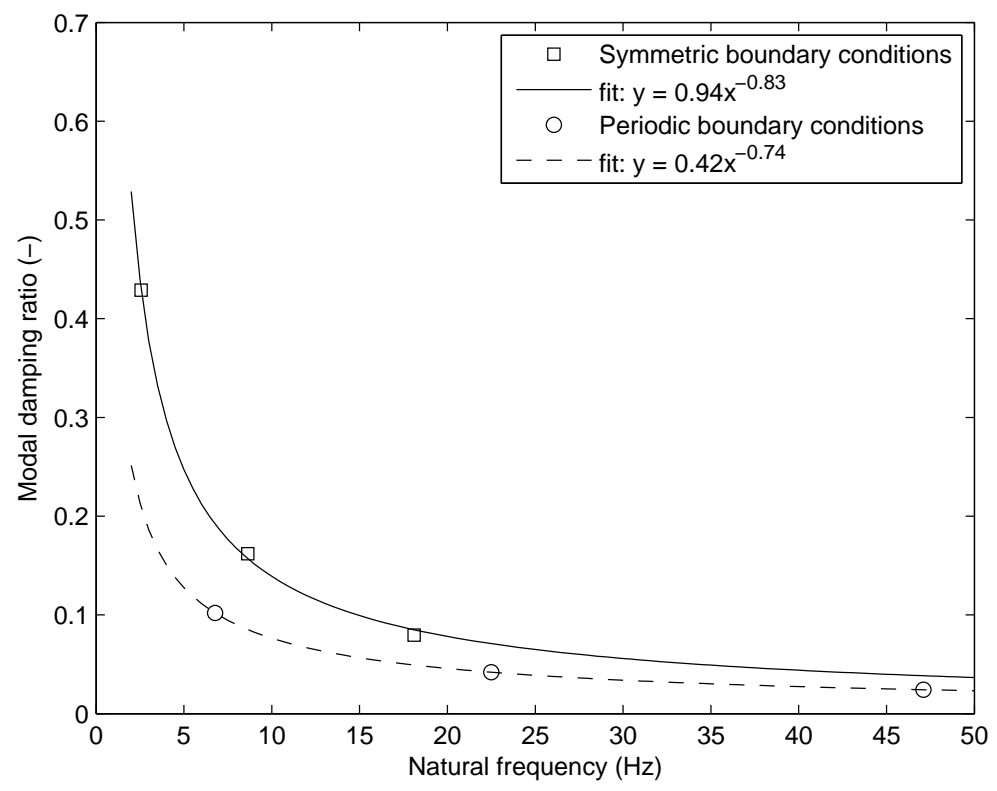

Figure 9: Modal damping ratio as a function of natural frequency for symmetric and periodic boundary conditions.

stagnation points and the other half stems from the coincidence of the periodic flow perturbation and the flow that goes around the moving tube.

The flow field for a flexible tube surrounded by 11 fixed tubes is similar to the flow field of the one with two surrounding tubes, but a larger amount of the flow perturbation is going round the tube instead of going through the periodic boundary conditions. Compared to the previous case, the periodic effect of the boundary conditions is a lot smaller. As a result, the confinement of the flow perturbation is higher (or less self-propelled from the motion of periodic repeats).

As the number of surrounding fixed tubes increases the frequencies of the modes in Table 5 decrease. The periodic boundary conditions have less effect when they are further away from the central cylinder and as a result the flow pattern changes to the one described above, which leads to a higher added mass. The modal damping will similarly increase with an increasing number of surrounding tubes. Computations with one extra layer of rigid tubes gave no significant changes.

Table 5: Vibration characteristics for a flexible cylinder surrounded by rigid ones.

\begin{tabular}{ccccccc}
\hline & $f_{1}(\mathrm{~Hz})$ & $\zeta_{1}$ & $f_{2}(\mathrm{~Hz})$ & $\zeta_{2}$ & $f_{3}(\mathrm{~Hz})$ & $\zeta_{3}$ \\
\hline 1 tube, symmetric & 2.56 & 0.429 & 8.64 & 0.162 & 18.1 & 0.0795 \\
1 tube, periodic & 6.78 & 0.102 & 22.5 & 0.042 & 47.1 & 0.0242 \\
3 tubes & 4.57 & 0.179 & 15.2 & 0.0700 & 31.8 & 0.0385 \\
12 tubes & 3.93 & 0.232 & 13.0 & 0.0866 & 27.3 & 0.0462 \\
Païdoussis (2004) & 3.8 & 0.182 & 12.6 & 0.0659 & 26.4 & 0.0361 \\
\hline
\end{tabular}

\subsection{Comparison with linear theory}

The analytic results are computed, as explained in Section 5.3, with the linear theory of Païdoussis (2004). The added mass coefficient used is again computed with the expression of Chen (1987), but with a modified equivalent 

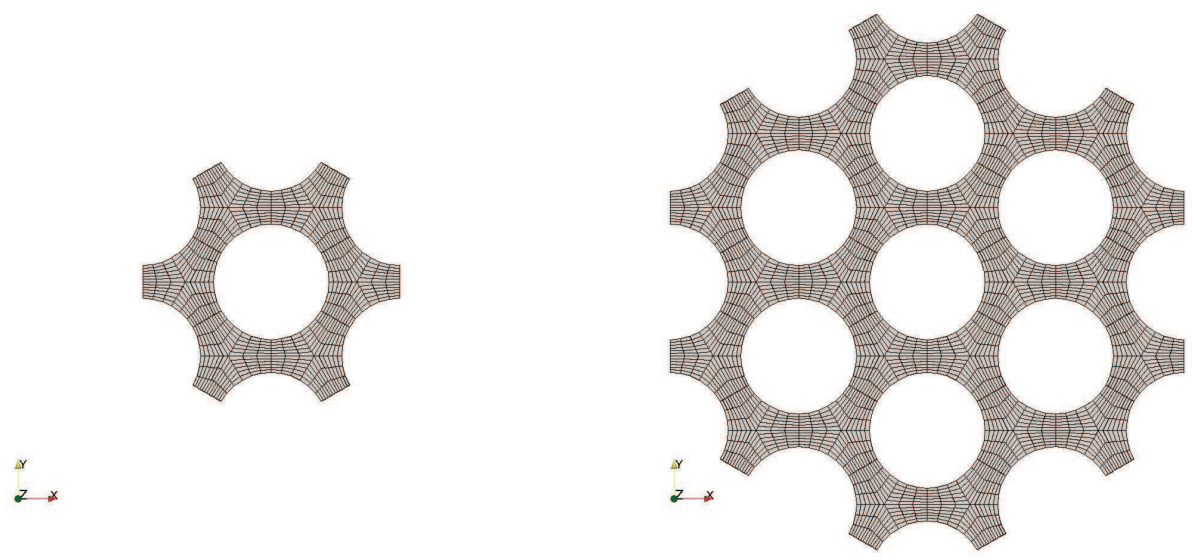

Figure 10: Computational mesh of the fluid for the case with 3 cylinders and 12 cylinders.

outer diameter, given by Au-Yang (2001):

$$
D_{e}=\left(1+P / 2 D_{o}\right) \cdot P \text {. }
$$

In the expression of Païdoussis (2004) three friction factors are appearing. The first of these friction factors is related to viscous damping in a quiescent flow, which can be computed from Chen (1987), with the modified equivalent outer diameter. The other friction factor, $C_{N}$ is often kept at a constant value between 0.004 and 0.03 . Based on experiments Blevins (1990) proposed a different formula for bundles with $P / D_{o}$ ratio of 1.33 , close to the $P / D_{o}$ ratio of 1.28 in this work:

$$
C_{N}=\left(\rho_{f} \cdot v \cdot D_{o} / \mu_{f}\right)^{-0.22}
$$

The resulting $C_{N}$ from this equation is 0.094 , significantly higher than common values. In the original formulation the value of the normal drag coefficient should equal the tangential drag coefficient. Experiments however indicate that $0.5<C_{N} / C_{T}<2$.

The value of the tangential friction coefficient has in this case only a minor effect: changing the value from 0.0028 (as computed by Equation 16) to 0.094 (equal to $C_{N}$ ) only results in a frequency and modal damping change of one percent. Therefore Equation 16 is used.

From Table 5 one immediately notices that the frequencies of the numeric case with 11 fixed surrounding tubes compare very well with the results of from the linear theory. The damping in the numeric case is higher than in the numeric simulation. This could mean that the normal drag coefficient should be even higher than the used value, as the $P / D_{o}$ ratio in this case is lower than 1.33 .

\section{Conclusions}

In this article a methodology for the determination of eigenmodes of a structure in a fluid flow is proposed. The methodology relies entirely on numerical fluid-structure interaction calculations, thus minimizing the empirical input, compared to existing theories.

This method has been validated with experiments available in literature (Chen and Wambsganss, 1972). Regarding the frequencies the calculations showed a very good agreement with the experiments. The modal damping ratios predicted by the numeric method agreed even better with the experimental damping ratios. The modal characteristics in these turbulent simulations were not very dependent on the molecular viscosity.

Finally, simulations of a cylinder in $\mathrm{Pb}-\mathrm{Bi}$ eutectic are performed with different boundary conditions. The periodic flow boundary conditions were the least confining boundary conditions and resulted in the highest frequencies and 

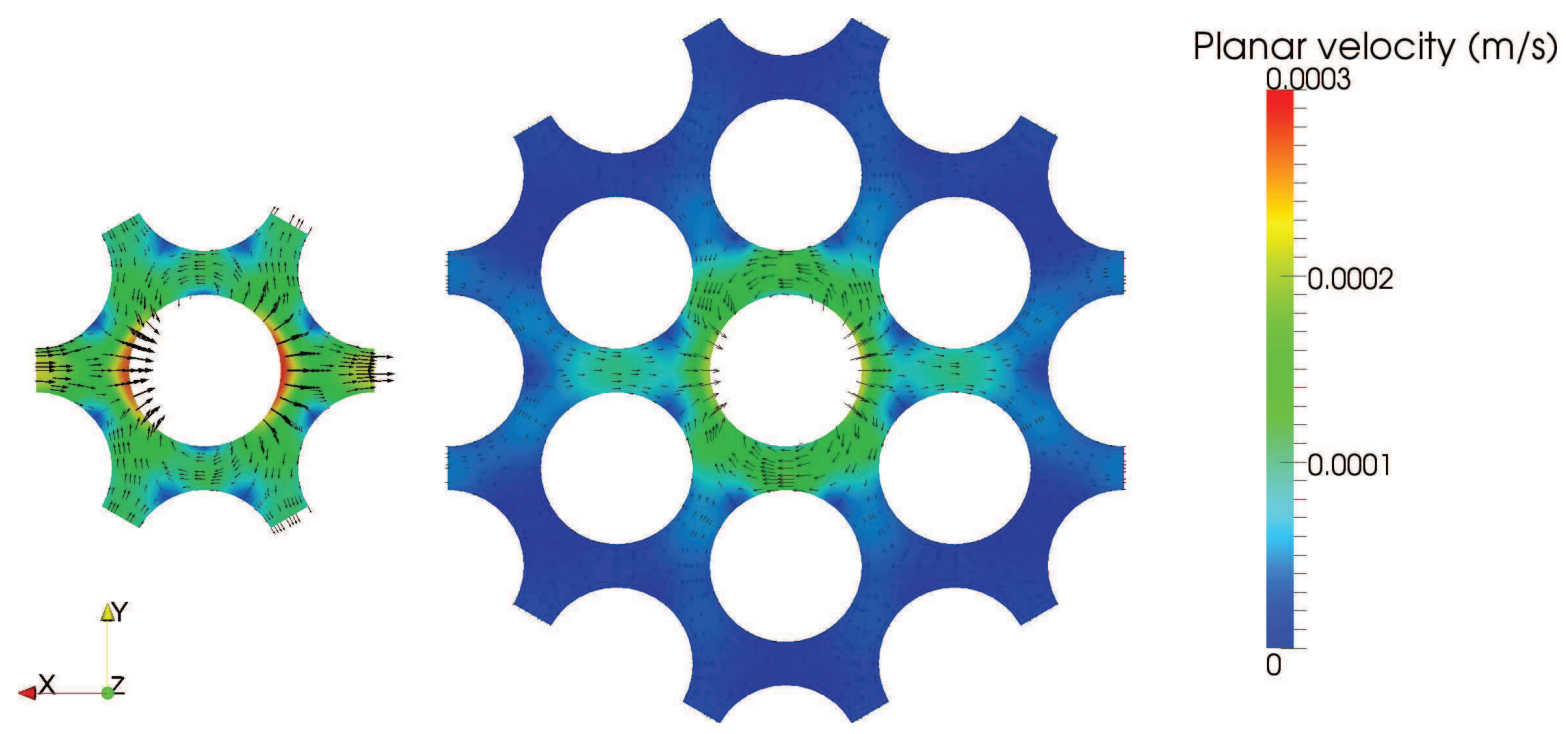

Figure 11: Flow pattern of the in-plane velocity $\sqrt{v_{x}^{2}+v_{y}^{2}}$ for first mode vibration after $t \approx T / 4$ for (left) 3 and (right) 12 tubes.

lowest modal damping ratios. The symmetric boundary conditions represented the most confining boundary conditions, while the simulations with multiple rigid cylinders surrounding one flexible tube where in between these two extremes. The frequencies agreed with frequencies predicted by current theories. The modal damping ratio showed some disagreement, which however improved when more relevant friction correlations were used in the existing theories.

\section{Acknowledgements}

The authors gratefully acknowledge the funding by the Research Foundation - Flanders (FWO), through the Ph.D. fellowship of Jeroen De Ridder and the postdoctoral fellowship of Joris Degroote.

Abderrahim, H. A., Baeten, P., De Bruyn, D., Heyse, J., Schuurmans, P., Wagemans, J., 2010. MYRRHA, a Multipurpose hYbrid Research Reactor for High-end Applications. Nuclear Physics News 20 (1), 24-28.

Au-Yang, K., 2001. Flow-induced vibration of power and process plant components. Professional Engineering Pub.

Belanger, F., Delangre, E., Axisa, F., Païdoussis, M. P., Mateescu, D., 1994. Dynamics of coaxial cylinders in laminar annular-flow by simultaneous integration of the navier-stokes and structural equations. Journal of Fluids and Structures 8 (7), 747-770.

Blevins, R. D., 1990. Flow-induced vibration. Van Nostrand Reinhold.

Chen, S. S., 1987. Flow-induced vibration of circular cylindrical structures. Hemisphere Pub. Corp.

Chen, S. S., Wambsganss, M. W., 1972. Parallel-flow-induced vibration of fuel rods. Nuclear Engineering and Design 18 (2), $253-278$.

de Langre, E., Païdoussis, M. P., Doaré, O., Modarres-Sadeghi, Y., 2007. Flutter of long flexible cylinders in axial flow. Journal of Fluid Mechanics $571,371-389$

Degroote, J., Bathe, K. J., Vierendeels, J., 2009. Performance of a new partitioned procedure versus a monolithic procedure in fluid-structure interaction. Computers and Structures 87 (11-12), 793-801.

Divaret, L., Cadot, O., Doaré, O., Moussou, P., Berland, J., 2012. Normal forces exerted upon a long oscillating cylinder in an axial flow. In: Proceedings of the 10th International conference on Flow-Induced Vibration. pp. 727-734.

Ersdal, S., Faltinsen, O. M., 2006. Normal forces on cylinders in near-axial flow. Journal of Fluids and Structures 22 (8), 1057-1077.

Facci, A. L., Porfiri, M., 2013. Analysis of three-dimensional effects in oscillating cantilevers immersed in viscous fluids. Journal of Fluids and Structures $38(0), 205-222$.

Fujita, K., Shintani, A., 2001. Axial leakage flow-induced vibration of the elastic rod as the axisymmetric continuous flexible beam. Journal of Pressure Vessel Technology-Transactions of the ASME 123 (4), 421-428.

Goyder, H., 2002. On the relationship between added mass and added damping. In: Proceedings of the 5th international symposium on fluid structure interaction, aeroelasticity, flow induced vibration and noise. ASME, pp. 745-753.

Hoerner, S. F., 1965. Fluid-dynamic drag: practical information on aerodynamic drag and hydrodynamic resistance. Hoerner Fluid Dynamics.

Inada, F., Hayama, S., 1990. A study on leakage-flow-induced vibrations. Part 1: Fluid-dynamic forces and moments acting on the walls of a narrow tapered passage. Journal of Fluids and Structures 4 (4), 395-412. 
Kestin, J., Sokolov, M., Wakeham, W. A., 1978. Viscosity of liquid water in range -8-degrees-c to 150-degrees-c. Journal of Physical and Chemical Reference Data 7 (3), 941-948.

Langthjem, M. A., Morita, H., Nakamura, T., Nakano, M., 2006. A flexible rod in annular leakage flow: Influence of turbulence and equilibrium offset, and analysis of instability mechanisms. Journal of Fluids and Structures 22 (5), 617-645.

Langthjem, M. A., Nakamura, T., 2007. Influence of swirl on the stability of a rod in annular leakage flow. Journal of Fluids and Structures 23 (2), 329-337.

Lighthill, M. J., 1960. Note on the swimming of slender fish. Journal of Fluid Mechanics 9 (2), 305-317.

Mateescu, D., Païdoussis, M. P., 1987. Unsteady viscous effects on the annular-flow-induced instabilities of a rigid cylindrical body in a narrow duct. Journal of Fluids and Structures 1 (2), 197-215.

Mateescu, D., Païdoussis, M. P., Bélanger, F., 1989. A theoretical model compared with experiments for the unsteady pressure on a cylinder oscillating in turbulent annular flow. Journal of Sound and Vibration 135 (3), 487-498.

Menter, F. R., 1994. 2-equation eddy-viscosity turbulence models for engineering applications. AIAA Journal 32 (8), $1598-1605$.

Meyer, L., 2010. From discovery to recognition of periodic large scale vortices in rod bundles as source of natural mixing between subchannels-a review. Nuclear Engineering and Design 240 (6), 1575-1588.

Mulcahy, T. M., Yeh, T. T., Miskevics, A. J., 1980. Turbulence and rod vibrations in an annular region with upstream disturbances. Journal of Sound and Vibration 69 (1), 59-69.

Nobile, F., Causin, P., Gerbeau, J. F., 2005. Added-mass effect in the design of partitioned algorithms for fluid-structure problems. Computer Methods in Applied Mechanics and Engineering 194 (42-44), 4506-4527.

Païdoussis, M. P., 1973. Dynamics of cylindrical structures subjected to axial flow. Journal of Sound and Vibration 29 (3), $365-385$.

Païdoussis, M. P., 2004. Fluid-Structure Interactions: Slender Structures and Axial Flow. Academic Press.

Païdoussis, M. P., Modarres-Sadeghi, Y., Semler, C., 2007. The nonlinear behaviour of a slender flexible cylinder pinned or clamped at both ends and subjected to axial flow. Computers and Structures 85 (11-14), 1121-1133.

Perotin, L., Granger, S., 1997. A linearized unsteady model for computing dynamics of cylindrical structures subjected to nonuniform annular flows at high reynolds numbers. Journal of Fluids and Structures 11 (2), 183-205.

Pettigrew, M. J., Taylor, C. E., 2003. Vibration analysis of shell-and-tube heat exchangers: an overview - Part 1: flow, damping, fluidelastic instability. Journal of Fluids and Structures 18 (5), 469-483.

Phan, N. C., Aureli, M., Porfiri, M., 2013. Finite amplitude vibrations of cantilevers of rectangular cross sections in viscous fluids. Journal of Fluids and Structures $40(0), 52-69$.

Rinaldi, S., Païdoussis, M. P., 2012. Theory and experiments on the dynamics of a free-clamped cylinder in confined axial air-flow. Journal of Fluids and Structures $28(0), 167-179$.

Sakuma, Y., Païdoussis, M. P., Price, S. J., 2008. Dynamics of trains and train-like articulated systems travelling in confined fluid-Part 1: Modelling and basic dynamics. Journal of Fluids and Structures 24 (7), 932-953.

Sinyavskii, V. F., Fedotovskii, V. S., Kukhtin, A. B., 1980. Oscillation of a cylinder in a viscous liquid. Soviet Applied Mechanics 16 (1), 46-50.

Sobolev, V., 2007. Thermophysical properties of lead and lead-bismuth eutectic. Journal of Nuclear Materials 362 (2-3), $235-247$.

Stokes, G. G., 1843. On some cases of fluid motion. Transactions of the Cambridge Philosophical Society 8, 30-37.

Wang, L., Ni, Q., 2009. Vibration of slender structures subjected to axial flow or axially towed in quiescent fluid. Advances in Acoustics and Vibration 2009. 